\title{
Research on the Online Teaching Mode of Chinese Characters as a Foreign Language Based on the Character-based Theory
}

\author{
Sun Man ${ }^{1, a}$, Qi Juanli ${ }^{2, b^{*}}$ \\ ${ }^{I}$ Department of Humanities Management, Shaanxi University of Traditional Chinese medicine, Qindu, Xianyang, \\ Shaanxi, China \\ ${ }^{2}$ Department of Literature and communication, Xianyang Normal School, Qindu, Xianyang, Shaanxi, China \\ a328701582@qq.com \\ b*qijunali2009@126.com
}

\begin{abstract}
Under the background of Internet, online teaching has become a trend. How to adapt to online teaching mode and how to combine theory with practice has become a problem for Chinese teachers. This paper, from the perspective of the "Character-based"theory, makes a thorough analysis of the unique advantages of the "Character-based"teaching method in the Chinese as a foreign language, and, in the light of the problems highlighted in the current online teaching of Chinese characters as a foreign language, explores the integration and innovation of the two methods, in order to provide teaching reference for on-line teaching of Chinese characters to foreigners, the implementation strategies of laying equal stress on handwritten Chinese characters and e-book Chinese characters are put forward.
\end{abstract}

Keywords: "Character-based"theory, Chinese as a foreign language, Chinese character teaching, Online teaching

\section{基于 “字本位” 理论的线上对外汉字教学模式探究 孙曼 ${ }^{1, a}$ 元娟莉 ${ }^{2 ， b * ~}$}

\footnotetext{
${ }^{1}$ 陕西中医药大学人文管理系, 秦都区, 咸阳, 陕西, 中国

2 咸阳师范学院文学与传播系, 秦都区, 咸阳, 陕西, 中国

a328701582@qq.com

b*qijunali2009@126.com
}

\section{摘要}

互联网大背景下, 线上教学已然大势所趋, 对外汉字教学如何适应线上教学模式, 如何将理论结合实际进行创 新, 成为对外汉语教师需要思考的一个问题。本文选取 “字本位” 理论的视角, 深入分析了 “字本位” 教学法 在对外汉语教学中的独特优势, 结合目前线上对外汉字教学所凸显的问题, 探索性地将两者融合创新, 提出了 手写汉字与电书汉字并重, 笔画部件的演示为主等实施策略, 以期为线上对外汉字教学提供教学参考。

关键词: 字本位, 对外汉语, 汉字教学, 线上教学

\section{1. 前言}

汉字教学是汉语作为第二语言教学最显著的特
点, 也是教学中的一大难点。相较于汉语语音、词汇、 语法教学研究, 汉字教学的研究仍然较为薄弱, 但已 经被越来越多的专家学者所重视。对于汉字教学应该 
遵循 “字本位” 还是 “词本位”, 应该“先语后文” 还 是 “语文共进”, 应该侧重 “工具汉字” 还是 “文化汉 字”, 学界都存在不同的声音, 还有学者提出了“电书 为主, 笔写为辅” 的汉字教学体系。时至今日, 后疫 情时代的线上教学模式 “异军突起”, 更对汉字教学 提出了新的挑战, 同时也为汉字教学模式创新打开了 新的突破口。

本文试从 “字本位” 理论为出发点, 讨论后疫情 时代线上教学模式下的对外汉字教学的模式创新及 应对策略。

\section{2. “字本位” 概念解析}

“字本位”这个概念在国内外语言学和教育学界 并不陌生, 目前学术界实际上存在着两种 “字本位” 之说。

从语言学的角度来说, 最早提出汉语 “字本位” 理论的是中国学者, 旨在将汉语研究与印欧语研究区 分开来。其中最具代表性的是徐通铭先生在《语义句 法刍议》(1990) 提出的 “字本位” 之说, 即从汉语本 体和汉语语法研究角度来看, 字是汉语的基本结构单 位, 而语素、词、句都是印欧语系的东西, 不适合汉 语。此外, 赵元任先生也看到了汉字在中文研究中的 独特性。他在《汉语词的概念及其结构和节奏》(1975) 一文中指出: “在说英语的人谈到 word 的大多数场合, 说汉语的人说到的是 “字”。这样说绝不意味着 “字” 的结构特性与英语的 word 相同, 甚至连近于相同也 谈不上。”

从汉语作为第二语言教学的角度出发, 法国著名 汉学家白乐桑先生在上个世纪 80 年代也提出了 “字 本位”之说, 又称为 “相对字本位”, 即 “以字带词” 的教学方法。其核心内涵是承认汉语具有“字”和“词” 两个语言教学单位, 主张汉语教学中字词兼顾, 从初 级阶段的以字带词, 过渡到中高级阶段的以词带字, 目前这一理论是法国现代汉语教学中的主流教学理 念。在国内, 也有很多学者支持 “字本位” 教学法, 吕必松先生就是 “字本位” 的坚定支持者, 他主张通 过研究汉语的组合规律来进行汉语教学, 目前已经出 了很多这方面的著作。

总的来说, 无论是在汉语本体研究中, 还是在汉 语学科教学研究中, 汉字都具有重要地位。与徐通铭 创立的 “字本位” 理论不同, 白乐桑先生提出的是基 于汉语二语教学论的 “字本位”, 所以本文采用的视 角是白乐桑先生的 “字本位” 理论。

\section{3. “字本位” 在对外汉字教学中的意义}

回顾中国的对外汉字教学，由于受到传统 “词本 位” 教学法影响, 初级阶段往往从拼音开始教学, 以 教词为主, 对于汉字只是教授简单的书写, 忽视了汉 字内部的逻辑性, 这就使得学生建立汉字形音义的连 接需要花费很长时间。白乐桑认为, 以适合表音文字
语言的教学法, 去教授表意文字的汉语, 必然会导致 把汉语复杂化的结果。陆俭明也支持 “字本位” 教学 法, 他在《我关于 “字本位” 的基本观点》一文中说:

“白乐桑先生提出的 “字本位” 汉语教学模式, 是一 项成功的创举。”

然而, 白乐桑先生的“字本位”教学法虽然在 1998 年就引入了中国, 也受到了汉语教学界的广泛关注和 重视, 但在实际教学环节仍然未得到充分运用, 在此 有必要重申一下“字本位”在对外汉语教学中的意义。

\section{1. “字本位” 突出汉语学习特点}

“字本位” 突出汉字在汉语学习中的重要性, 是 符合汉语自身特点的。

首先，汉字是表意文字。相对印欧语系的表音文 字, 汉字是 “不透明” 的, 不懂汉语的人往往不能根 据汉字字形判断字的读音。另外, 汉语语素以单音节 为主, 语素和 “字” 基本上是重合的, 这就说明汉字 的语义透明度很高, 可以说大大超过了表音文字。可 见, 汉字的形音义联结方式与印欧语系的语言非常不 同, 在学习方法上需要区别对待。

其次, 中国自古就有集中识字的传统, 以识字作 为汉语学习的基础, 正是中国传统语文教学的一贯理 念。那么将其应用于对外汉字教学中亦不足为奇, 甚 至更为合理。

\section{2. “字本位” 强调汉字教学地位}

“字本位”强调了汉字在对外汉语教学初级阶段 中的重要地位。在汉语作为第二语言教学这一学科的 发展过程中，一直存在着 “词本位” 与 “字本位”之 争, 前者坚持词是唯一的汉语语言基本教学单位, 后 者则认为汉语既有词又有字这两个基本教学单位。在 “词本位”盛行的教学形势下, “字本位” 无疑是将汉 字教学的地位提高到了前所未有的高度。

因此, 在 “字本位” 指导下的教学自然更重视初 期的汉字教学。

\section{3. “字本位” 符合学生认知规律}

“字本位” 提倡初期先学基础汉字, 然后从 “以 字带词” 到 “以词带字”, 从必然王国到自由王国, 循 序渐进地习得汉语。

以 “字本位” 的汉字教学按照字频和构词能力选 字, 在选字构词的基础上, 形成句子最后组合成语篇。 在讲解过程中, 注重从汉字的字源、笔画、部首以及 部件来教授汉字, 分析汉字的理据性, 这一做法符合 学生的记忆和认知规律, 尤其对于以分析性语言为母 语的外国学生, 分析拆解汉字的方法能够帮助他们更 有效地记忆汉字并培养学习兴趣。 


\section{4. “字本位” 指导教学科学有效}

《汉语语言文字启蒙》是白乐桑于 1989 年与张 朋朋合编的汉语教材，该教材充分体现了 “字本位” 的教学理念。这本教材高度重视字频和词频, 精选了 使用率最高的 400 个汉字进行编写, 每三篇课文后都 有一篇 “滚雪球” 文章以加强字的复现率, 学生可以 通过已学的字词猜出新词的意义。

1990 年至今, 巴黎第七大学中文系都在使用这本 教材, 法国前总统德斯坦学习中文时也采用了这本教 材, 可见, “字本位” 理念对于指导外国人学习汉语十 分可行并且成效显著。

\section{4. 线上对外汉字教学存在的问题}

如今, 线上教学已然是一种主流的教学模式, 习 惯了传统线下教学的教师和学生, 必然会在适应线上 教学的过程中出现一些问题。笔者认为造成这些问题 的背后原因, 是仍然沿用了传统教学模式的思路, 缺 乏针对线上教学模式的创新。总的来说, 线上对外汉 字教学的具体问题可以归纳为以下五点:

第一，无法实现临场监控汉字书写。笔者发现留 学生更倾向用打字的方法代替汉字书写, 即使是面对 面的情况下, 除非是在线下教学要求学生手写汉字, 否则他们很容易因为汉字难写或者忘记笔顺等原因 而放弃书写, 如果在线上教学, 对学生而言打字更方 便，则很有可能完全放弃汉字书写的学习。

第二, 线上板书演示不易操作。由于线上教学只 能使用软件工具模拟 “笔” 来书写汉字, 所以在书写 的熟练度及美观度上都不如真实情境下的笔, 而且因 为对使用鼠标写字不熟悉, 甚至会导致书写的偏误, 对学生造成错误的引导。

第三，网上学习资源良莠不齐。目前网络上对于 汉字的笔画笔顺知识仍然存在争议, 对于一些常见的 字, 如 “母” “火” 等字的笔顺, 就连母语者也未必能 按正确的笔顺书写。在一些 APP 中还出现了笔画数不 正确的情况，如 “这” 字的 “之” 是 3 笔，有的 APP 将“之”写成了 4 笔。这些情况都很容易误导学习者。

第四, 实时监管课堂有难度。虽然线上教学技术 能实现实时视频、实时语音的功能, 但从目前线上教 学的情况来看, 还是以老师开摄像头及麦克风为主, 学生一般只以打字的方式与老师进行互动。因此教师 难以通过网络实时观察到学生的听课反应, 难以及时 根据学生反应调整教学策略。

第五, 网络存在不稳定因素。网络因素是不可控 性最多的因素, 因为学生和教师身处不同地区和国家, 难免会出现网络卡顿或者延迟问题, 对教学进程造成 一定程度的影响。

\section{5. “字本位” 与线上教学模式的融合创新及 运用策略}

“字本位” 理论提出后, 很多教师将这一理论应 用到了传统线下教学中, 还并未见于线上教学模式中 的研究。因此, 笔者试从 “字本位” 的视角, 讨论线 上汉字教学模式的创新及实施策略。针对这一问题, 笔者提出了以下五点建议:

\section{1. 手写汉字与电书汉字并重}

互联网大背景下, 汉字作为书面语交流的工具出 现了不同的呈现方式, 即手写汉字和电书汉字。其中 手写汉字是传统教学中的主要教学内容, 而如今网络 已经渗透到日常生活的方方面面, 很多活动场景已经 不局限于现实, 而更多地转移到网络世界, 这就亟需 网络参与者掌握电书汉字, 即使用键盘输入汉字的能 力。因此, 考虑到培养学生用汉字进行交际的能力, 应将电书汉字纳入汉字教学中。

但这并不意味着手写汉字的时代已经过去了, 汉 字教学的一大重点就是 “写”, 只有在学生亲身体会 “写” 的过程之后, 才更加能感悟到汉字的形态美、 结构美和书法美, 进而更深刻地领会到中华文化的博 大精深, 由此汉字教学还能够进一步扩展为文化教学 的一部分, 而这个功能是电书汉字教学无法完全实现 的。

所以, 我们在考虑 “字本位” 的前提下, 兼顾手 写汉字和电书汉字, 能够缓解学生对汉字的畏难情绪, 同时又做到不偏废汉字的书写, 而且更能高效地将汉 字应用于日常生活之中。

\section{2. 笔画和部件展示为主, 鼠标板书为辅}

在讲解汉字的结构和笔顺时, 由于不方便使用传 统板书, 教师应充分利用网络资源, 以笔画和部件展 示为主, 手写板书的演示为辅。

具体来说, 在教独体字时, 可以通过单独演示笔 画的动画, 字源和造字法知识帮助学生记忆; 教合体 字时, 不仅要用到上述方法, 还要结合拆分部件的方 法演示合体字。周小兵在《对外汉语教学入门》一书 中总结了以部件为中心的汉字教学, 是符合汉字认知 和习得规律的。在汉字习得研究中, 相当一部分汉字 偏误都是部件的偏误, 而且随着汉语水平的提高, 部 件偏误的比例会越来越大。通过归纳部件来学习汉字, 可以帮助学生分析汉字结构, 强化部件的位置, 区分 相似部件, 归纳同音字等。教师应该利用生动有趣的 网络资源, 下载一些部件碎片和动画, 将其插入在讲 解过程和练习环节或者游戏环节之中, 利用网络传播 的优势, 将汉字生动地呈现在学生面前。

除此之外, 用鼠标板书演示可以适当加入, 作为 临时的提示和即兴讲解的辅助。 


\section{3. 甄选学习 $A P P$, 推荐权威网站}

目前手机应用市场上有不少针对汉语和汉字学 习的 APP, 一方面说明汉语文化真的热起来了, 另一 方面, 由于监管不严, 其中难免有鱼目混珠的 APP, 教 师和学生需要对其内容和质量做一甄别。

笔者发现留学生也会在课下主动下载一些汉语 学习 APP, 针对这些 APP, 教师应该具有敏锐的甄别 能力。由于留学生不具备足够充分的汉语知识, 对于 网上学习资源缺乏准确的判断, 如果某些资料的内容 有误, 很有可能误导学习者。所以, 笔者建议教师积 极了解学生在课下的学习情况, 主动研究各种汉语学 习 APP, 甄选出几款可供参考的 APP, 学生可选择性 下载; 或者由教师统一指定一款学习 APP, 这样不仅 便于学生课下学习, 而且便于学生们交流使用心得。

此外, 教师还可以向学生推荐一些权威的汉语学 习网站。比如网络孔子学院、中国网 (Learning Chinese )、朗朗中文、魔方汉语、Games Learn Chinese 等。其中有的网站有汉字游戏, 教师也可以 在线上汉字教学时加以运用。此外还有一些汉字游戏 APP 以及小程序, 教师也可以甄选一些出来推荐给学 生。

\section{4. 引导学生制作 “字词树”, 提高学生的课 堂参与度}

“字词树”是以一节课的 “核心字”为树的 “主 干”, 以 “核心字” 衍生出的几个常见高频词为树的 “枝干” 展开的, 可根据学生水平、教学大纲及教学 内容做适当调整。初级阶段无需添加过多 “枝干”, 随 着教学内容的逐渐丰富, 每一棵 “核心字” 的树也会 逐渐成长, 这与白乐桑 “滚雪球” 式的教学方法异曲 同工。制作 “字词树” 能够发挥学生的主观能动性和 想象力, 提高学生的课堂参与度。制作过程以学生为 中心, 教师做主导, 根据教学内容及学生水平, 适当 拓展 “字词树” 的内容。

“字词树” 的教学形式遵循以 “学生为中心” 的 教学原则, 既提高了学生参与课堂的积极性, 又增加 了学习汉字的趣味性, 让学生从中获得了学习汉字的 成就感, 进一步培养了学生对汉语学习的兴趣。此外, “字词树” 的形式也便于教师对课程做阶段性的总结 和评价。

\section{5. 利用软件的互动功能辅助监控, 作业的呈 现形式求新求变}

由于在线上教学, 教师不能实地了解到学生的个 人特点、学习风格及学习效果等情况, 就要借助各种 软件的互动功能。比如在企业微信中, 教师能够在群 组中发起问卷调查, 在课上或者课下实时跟踪了解学 生的学习情况, 教师也能够在企业微信中监控到有多 少学生阅读并下载了资料。因此, 能够发掘教学软件
的功能并充分利用到教学中, 也是线上教学的教师需 具备的一种能力。

在作业的呈现方式上，由于线上教学不方便提交 纸质作业, 教师应考虑布置多元化的作业形式。比如, 在学习了一节课的汉字之后, 教师为了考察每个人书 写汉字的情况, 可以布置短视频形式的作业, 要求学 生将书写汉字的过程录下来, 提交在学习群组中, 或 者单独发给教师。为了考察学生的笔画笔顺掌握情况, 可以图片的形式呈现。在完成了一个阶段的学习之后, 教师可以要求学生提交这一阶段所做的 “字词树” 作 为阶段性考核。如果教师指定学生使用同一款汉字游 戏 APP, 也可以将游戏闯关记录作为一个考核因素。 总之, 线上教学模式下作业的呈现形式应求新求变, 注重趣味化和多样化。

\section{6. 结论}

如今互联网高速发展, 线上教学已然成为一种重 要的教学模式, 而新冠疫情的爆发更加推动了线上教 学的发展, 其优势与劣势也愈发凸显。而关于线上教 学法的探讨尚有不足, 可谓是时代向我们提出的共同 课题。笔者从线上对外汉字教学考虑, 基于白乐桑先 生的 “字本位” 教学法, 理论结合实际, 提出了若干 思考与建议, 希望对对外汉语线上教学的理论与实践 贡献微薄之力。

\section{REFERENCES}

[1] Huang Borong,Liao Xudong.(1997)Standard Chinese. Higher Education Press,Beijing.

[2] Liu Xun.(2014) An introduction to the pedagogy of Chinese as a foreign language .Beijing Language and Culture University Press,Beijing.

[3] Bai Lesang.(2017) Cross-cultural Chinese pedagogy. Encyclopedia of China Publishing House,Beijing.

[4] Zhou Xiaobin.(2017) Chinese as a foreign language.SYSU Press, Guangzhou.

[5] Bai Lesang.(2018)Monism or dualism: fundamental differences and obstacles in the ontological epistemology of Chinese second language teaching. Teaching and research in Chinese, $04: 1-11+24$.

[6] Bai Lesang.(2019) Chinese teaching method with words . Melvin, 02:43-50

[7] Lu Jianming.(2011) My basic view on "Character standard". Language Science,10(03):225-230

[8] Liao Kai.(2021) Problems and countermeasures of online Chinese character teaching in TCFL . Literary Education, 05:148-150.

[9] Zhou Yonghua,Li Shanlin.(2018) The application of "Character standard"theory in Chinese character teaching. Educational observation,7(12):16-18 